\title{
Fusing Multi-scale and Multi-modal 3D Imaging and Characterization
}

\author{
Arno Merkle ${ }^{1}$, Leah L. Lavery ${ }^{1}$, Jeff Gelb ${ }^{1}$ and Nicholas Piché ${ }^{2}$ \\ ${ }^{1 .}$ Carl Zeiss X-ray Microscopy, Inc., Pleasanton, CA, USA \\ ${ }^{2}$ Object Research Systems, Montreal, Quebec, Canada
}

Three-dimensional X-ray microscopy (XRM) has emerged as a powerful interior imaging technique that obtains information from a range of materials under a variety of conditions and environments. Recently, laboratory-based X-ray microscopes have demonstrated tomographic datasets with resolution down to $50 \mathrm{~nm}$ for investigation across a great span of sample dimensions from the nanoscale to the mesoscale. [1-3] It has been used to study a wide spectrum of materials from carbonate rocks to murine brains, including hierarchical structured materials. [4] This talk will present the current state of the art in laboratory X-ray microscopy at various length scales and multi-modal capabilities for XRM-SEM combined image visualization, transformation, manipulation, and analysis using commercial software package from Object Research Systems (ORS).

X-ray microscopy (XRM) is currently evolving into standard practice for many disciplines and the technique is fueled by the historical developments that have taken place in the synchrotron facilities worldwide. Using reflective capillary condenser lenses coupled with diffractive Fresnel imaging objectives, beamlines in some facilities are now pushing the $10 \mathrm{~nm}$ resolution levels. [5] Laboratory instrumentation has grown out of these developments, and laboratory X-ray microscopes are now able to achieve resolutions on the order of $50 \mathrm{~nm}$, using analogous optical architectures to those in the synchrotrons (with obvious source differences). Commercially available nano-XRMs, such as the ZEISS Xradia Ultra series, are transitioning this technology into the central laboratories (Figure 1), enabling unprecedented high-resolution studies of soft and hard materials alike without the need for synchrotron radiation. The primary driver for this need is to focus on correlative and time-dependent (4D) experiments.

Micron-scale X-ray tomography is also commonplace in synchrotrons, but traditional laboratory micro-and nano-CT instrumentation has been lagging behind the synchrotron developments. This is due largely to the system geometry most often used by laboratory micro- and nano-CT suppliers, which relies on a standard laboratory X-ray source producing a beam with large angular divergence to deliver high geometric magnification. This approach can work well for small sample sizes, but, in practice this imposes physical sample size limitations due to the physics of such a design. By implementing a novel $\mathrm{X}$-ray detection system, mirroring the designs in place at synchrotron micro- and nano-TXM beamlines, sub-micron resolution is now routinely achievable in the laboratory as well with the ZEISS Xradia Versa series across a large range of sample sizes. The lowered geometrical restrictions are met with a tunable contrast enhancement mechanism, which combines optimized scintillation technology for both absorption and propagation phase contrast, for a uniquely flexible laboratory X-ray microscope platform for modern in-situ and 4D studies on a wide variety of sample types. [1]

XRM provides not only 3D image visualization, but a 3D dataset, which requires advanced analysis and robust data management for accurate segmentation and volumetric quantification of material properties. These datasets have been combined with subsequent characterization using 3D FIB-SEM techniques, to 
extend structure investigation to the single nanometer length scales. Manipulating and analyzing these large data stacks requires high display speeds and the ability to handle large datasets, for which a commercial software package from Object Research Systems (ORS) has been implemented.

In this presentation, we will explore the multi-modal capabilities for XRM-SEM combined image visualization, transformation, manipulation, and analysis using standard PC hardware and GPU shader technology with a high-degree of accuracy. A data analysis workflow will be presented for visualization and analysis of a multi-scale and multi-modality 3D dataset on $\mathrm{Cu}-\mathrm{Al}$ eutectic alloy (Figure 3). The same region of interest of the alloy was imaged in 3D via XRM (ZEISS 520 Versa and 810 Ultra) and FIB-SEM (ZEISS Auriga), using a voxel size of 1 micron down to $2 \mathrm{~nm}$. Additional workflows using ORS to analyze XRM datasets will be presented for volumetric characterization of shale and 3D interior structure of an orchid.

\section{References:}

[1] A. P. Merkle and J. Gelb, Microscopy Today, 21, (2013), p. 10.

[2] A. Tkachuk, F. Duewer, H. Cui, et al., Z. Kristallogr. 222, (2007), p. 650.

[3] E. Maire and P. Withers, Quantitative X-ray tomography, International Materials Review, 59 (2014).

[4] H. Sai, K. W. Tan, K. Hur, et al, Science, 341, (2013), p. 530. [5] Y. Feng, et al., J. Vac. Sci.

Technol. B, 25, (2007), p. 2004.

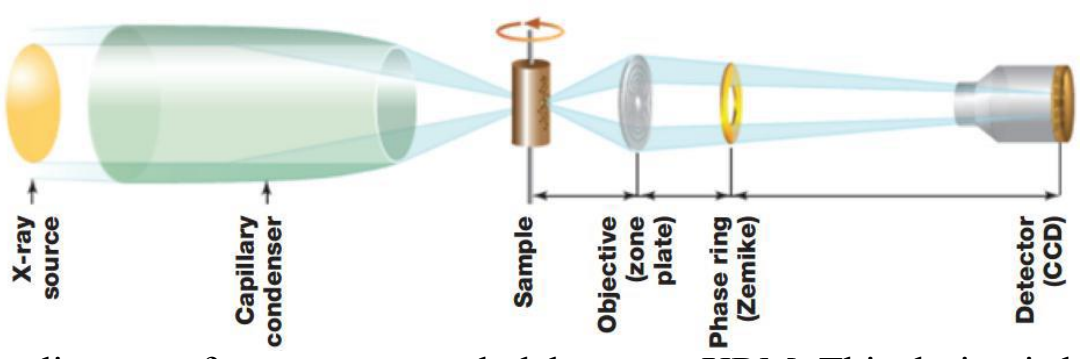

Figure 1. Schematic diagram of a true nano-scale laboratory XRM. This design is based on synchrotron developments, using X-ray focusing optics to achieve high spatial resolution.

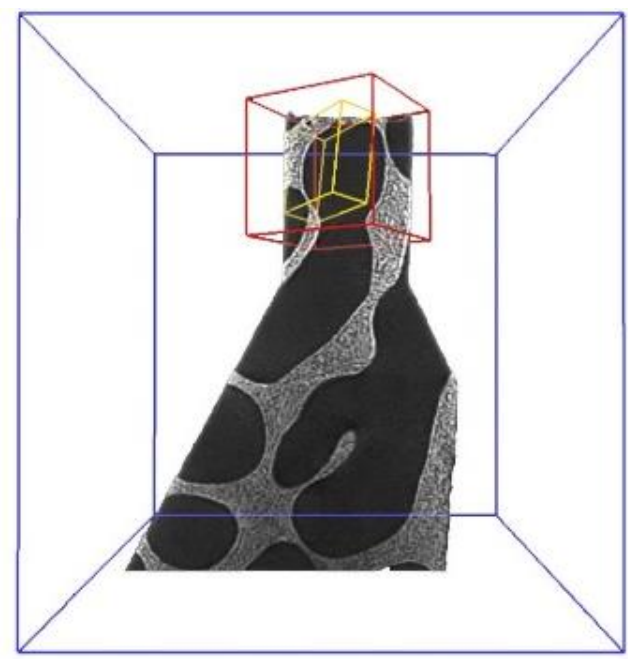

Figure 2. Cu-Al eutectic alloy. The same region of interest of the alloy (designated by the cube volumes) was imaged in 3D via x-ray microscopy (ZEISS 520 Versa and 810 Ultra) and FIB-SEM (ZEISS Auriga). using a voxel size of 1 micron down to $2 \mathrm{~nm}$. Sample courtesy of B. Patterson, Los Alamos National Laboratory. 